\title{
Anthrax: The Investigation of a Deadly Outbreak
}

\author{
by Jeanne Guillemin \\ University of California Press, \$19.25, 321 pp. \\ ISBN: 0-520-22204-0, 1999
}

\author{
REVIEWED BY \\ DONALD A. HENDERSON \\ Center for Civilian Biodefense Studies \\ The Johns Hopkins University \\ Baltimore, Maryland 21202, USA \\ Email:dhenders@jhsph.edu
}

On 2 April 1979, a small cloud of anthrax spores was accidentally released from a bioweapons production facility in Sverdlovsk, a Soviet city in the Ural Mountains. It drifted slowly southeast over a portion of the city's southern suburbs-invisible, unrecognized. Two days later, the first victims fell ill. Over the following six weeks, some 100 people developed the disease and at least 70 died as a result of infection acquired as far as three miles downwind from the release point of the spores. This was the first and only known epidemic of inhalation anthrax. Calculations indicate that the quantity of spores released may have weighed less than one gram.

Anthrax is one of the most deadly of the potential biological weapons and the agent most universally favored by those desiring to produce such weapons. In nature, it is mainly a disease of grazing animals, which acquire infection from contaminated soil. Natural infections in man result from contact with animals or animal products and usually cause an ulcer of the skin that is readily treated with antibiotics. There are occasional small outbreaks of gastrointestinal disease when undercooked, contaminated meat is consumed. However, inhalation anthrax is an exceedingly rare but grave form of the disease that kills up to $80 \%$ of its victims. From the onset of infection to death is usually no more than two to four days. Antibiotics are of little benefit in treatment unless administered very soon after the first symptoms occur.

In her book Anthrax, Jeanne Guillemin provides the only detailed account of the appalling Sverdlovsk epidemic, an event that could be repeated

on a far larger scale were anthrax spores in an aerosol form to be disseminated today by a terrorist. The book is structured around the evolving investigation of the event led by Harvard biologist Mathew Meselson, assisted by the author and several Russian and American scientists. The format is reminiscent of Berton Roueche's New Yorker tales of epidemic sleuthing.

From the time of occurrence of the epidemic in 1979, Russian scientists attributed its cause to the consumption of contaminated meat. However, rumors provided by emigrés suggested otherwise to US intelligence analysts. In the interest of defusing suspicions about Russian biological weapons developments, Meselson had repeatedly pressed Russian officials and scientists for a full and open investigation of the incident. Finally, in June 1992, permission was granted for an American team to visit Sverdlovsk. Russian Ministry of Health officials persisted in their assertion that the cause was contaminated meat and provided little essential data. The challenge to the team was that of investigating an epidemic more than a decade old, one in which the clinical records had been confiscated by the KGB and in which most victims had died of the disease.

At the outset, an essential breakthrough occurred with the discovery of two pathologists who had completed numerous autopsies, 42 of which they considered to be classical inhalation anthrax. Fortunately, the specimens had been overlooked by KGB officials seek-

\section{The Paradox of Sleep}

\section{by Michel Jouvet \\ The MIT Press, \$26.00, 211 pp. ISBN: 0262100800, 1999}

\section{Reviewed by Allan Hobson \\ Harvard Medical School \\ Department of Psychiatry \\ Laboratory of Neurophysiology 74 Fenwood Road \\ Boston, Massachusetts 02115, USA}

When nearing the end of their careers, many scientists indulge themselves in writings that reveal their distinctly unscientific views on such heady matters ing to confiscate all relevant data. An American pathologist on the team was able to confirm that they were typical of inhalation anthrax. Gradually, thereafter, the story unfolded as the author, assisted by Russian counterparts, interviewed relatives of many of the survivors and systematically assembled, piece by piece, considerable information about the epidemic.

Perhaps the most important finding was the discovery that the incubation period for inhalation anthrax was not two to six days, as experimental animal studies had shown and as standard textbooks proclaimed. Rather, it was two to at least forty-five days. The implication of this finding is that after discovery of an epidemic, there would be sufficient time to administer life-saving antibiotic prophylaxis to prevent disease in up to $75 \%$ of the potential victims.

Scientists and policy-makers alike will be disappointed that the author did not devote more time and effort to the analysis and elucidation of the trove of epidemiological information that apparently was collected. And what might have been a riveting epidemiological detective story drifts too often into a rambling travelogue with extended irrelevant digressions.

The book should be required reading for anyone seriously concerned about biological terrorism, but it is difficult to recommend it to others with a more general interest in epidemiology or cultural anthropology.

as the creation of the cosmos, the origin of man or the transmigration of the soul. In the case of neuroscientists, a favorite senile indulgence is the mind-body problem. In a paradoxical way, Michel Jouvet's The Paradox of Sleep is a book of this genre.

Yes, Jouvet's musings on the functions of sleep are highly speculative. Yes, they are philosophically confused. And yes, they are wildly uncritical. But where others like Charles Sherrington, Santiago Ramon y Cajal and John Eccles have been tempted to give up on the body in favor of the mind, or even the spirit, Jouvet remains passionately committed to biology. The result is a highly readable account of a highly 